\section{FAST OR SLOW: DIAGNOSTIC CHALLENGES IN OCULAR AND CEREBRAL NOCARDIOSIS}

\author{
Annaleise R. Howard-Jones $^{1,2}$, Heeral Thakkar ${ }^{3}$, \\ Vidthiya Menon ${ }^{2,3}$ \\ ${ }^{1}$ NSW Health Pathology-Nepean, Nepean Hospital, Kingswood, \\ NSW, Australia; ${ }^{2}$ Faculty of Medicine and Health, University of \\ Sydney, Camperdown, NSW, Australia; ${ }^{3}$ Department of \\ Microbiology \& Infectious Diseases, Nepean Hospital, \\ Kingswood, NSW, Australia
}

Manifestations of Nocardia infections are diverse due to the ability of the organism to disseminate to almost any organ. ${ }^{1}$ Cerebral infection is not uncommon, particularly with Nocardia paucivorans, ${ }^{2}$ but ocular disease is rare. ${ }^{3}$

We present the case of a 63-year-old immunocompetent gentleman with disseminated nocardiosis. Though his initial presentation with shoulder pain and malaise led promptly to the diagnosis of $N$. paucivorans septic arthritis from tissue biopsy, the diagnosis of multiple cerebral abscesses, left eye endophthalmitis with subretinal abscess and hilar lymphadenopathy followed new evolution of central nervous system signs and an elicited history of a subacute left eye floater.

Treatment with intravenous meropenem, linezolid and trimethoprim/sulfamethoxazole for 2 weeks followed by intravenous ceftriaxone and oral linezolid and moxifloxacin was complemented by intravitreal amikacin. He was transitioned to dual agent oral trimethoprim/sulfamethoxazole and moxifloxacin therapy after 8 weeks following clinical and radiological improvement at all sites.

Ocular nocardiosis presents unique diagnostic and management challenges due to considerations for biopsy, intravitreal antibiotics and/or vitrectomy and the importance of intra-ocular penetration of the systemic antibiotic regime.

This case demonstrates that, although immunosuppression is a known risk factor for severe nocardiosis, disseminated disease may also be seen even in immunocompetent patients. ${ }^{1}$

\section{References}

1. Beaman BL, Beaman L. Nocardia species: host-parasite relationships. Clin Microbiol Rev 1994; 7: 213-64.

2. Hammoud M, Kraft C, Pulst-Korenberg J, et al. Disseminated Nocardia paucivorans infection in an immunocompetent host. Infection 2014; 42: 917-20.

3. de Silva T, Evans C, Mudhar HS, et al. Isolated endogenous endophthalmitis secondary to Nocardia spp. in an immunocompetent adult. J Clin Pathol 2006; 59: 1226.

\section{AN UPDATE ON COVID-19 SEROLOGY}

Paul M. Kinsella, Theo Karapanagiotidis, Suellen Nicholson, Deborah A. Williamson

Royal Melbourne Hospital, Melbourne, Vic, Australia

Since the onset of the SARS-CoV-2 pandemic, diagnosis of acute infection has predominantly been achieved using nucleic acid amplification. However, serological testing methods have retained utility in specific clinical and public health settings. The technology used in these assays has evolved over time from the first lateral flow assays to high-throughput commercial enzyme immunoassays and surrogate virus neutralisation tests. Here, we provide an overview of the clinical and public health utility of SARS-CoV-2 serological testing and explore future applications of these assays to aid in management of the SARS$\mathrm{CoV}-2$ pandemic. In addition, we provide evidence for the use of novel approaches to serological testing, including testing of dried blood spots.

\section{FEVER, EOSINOPHILIA AND ABDOMINAL PAIN}

Kwee Chin Liew $^{1}$, Kim Yeoh ${ }^{1}$, Cecillia Lai ${ }^{1}$, Eloise Williams ${ }^{1}$, Harsha Sheorey $^{1}$, Siddhartha Mahanty ${ }^{2}$

${ }^{1}$ Department of Microbiology, Royal Melbourne Hospital at The Peter Doherty Institute for Infection and Immunity, Melbourne, Vic, Australia; ${ }^{2}$ Department of Infectious Diseases, Royal Melbourne Hospital, Melbourne, Vic, Australia

We describe a case of liver hydatid cyst in a 34-year-old female who presented to hospital with a 4-day history of epigastric pain and fever. Her past medical history was unremarkable. She was born in Mumbai, India, and migrated to Australia in 2015. She did not have farm contact and/or wildlife exposures. Her white cell count was elevated $15.3 \times 10^{9} / \mathrm{L}$, with a predominant eosinophilia $4.1 \times 10^{9} / \mathrm{L}$, CRP $256 \mathrm{mg} / \mathrm{L}$, AST $32 \mathrm{U} / \mathrm{L}$, ALT $59 \mathrm{U} / \mathrm{L}$, ALP $195 \mathrm{U} / \mathrm{L}$ and GGT $152 \mathrm{U} / \mathrm{L}$. Echinococcus granulosis serology (EIA) was low positive. Abdominal ultrasonography and computed tomography showed a $13.3 \mathrm{~cm}$ liver lesion involving most of the left lobe of the liver with a surrounding hypodense thickened periphery, possibly with some haemorrhage. The patient was treated with albendazole $400 \mathrm{mg}$ BD for 6 weeks before her elective laparotomy, deroofing of the hydatid cyst, removal of cyst contents, followed by cetrimide infusion into the cyst as a scolicidal agent. Echinococcus granulosis hooklets were seen on direct microscopy and trichome stain. Degenerated protoscolices were seen on histology. The patient was treated with 3 months of albendazole post-operation as the hydatid cyst involved all three hepatic veins and could not be completely resected. Her abdominal pain, eosinophilia and inflammatory markers improved post treatment.

\section{FIRST REPORTED HUMAN DEATH DUE TO INVASIVE ASPERGILLUS FELIS INFECTION IN AN IMMUNOCOMPETENT HOST}

Adam G. Stewart ${ }^{1,2,3}$, Patrick N. A. Harris ${ }^{1,3}$, Claire Heney ${ }^{3}$
${ }^{1}$ Centre for Clinical Research, Faculty of Medicine, The
University of Queensland, Royal Brisbane and Women's
Hospital Campus, Brisbane, Qld, Australia; ${ }^{2}$ Department of
Infectious Diseases, Royal Brisbane and Women's Hospital,
Brisbane, Qld, Australia; ${ }^{3}$ Central Microbiology, Pathology 